\title{
ON RECIPROCAL POLYNOMIALS WITH ZEROS OF MODULUS ONE
}

\author{
LÁSZLÓ LOSONCZI
}

Abstract. The purpose of this paper is to characterize reciprocal polynomials all of whose zeros are on the unit circle. Using this characterization we give bounds for the coefficients of such polynomials and show how other necessary conditions (inequalities for the coefficients) can be obtained from the characterization theorem.

Mathematics subject classification (2000): 30C15, 12D10, 42C05.

Key words and phrases: reciprocal polynomial, zeros, unit circle.

\section{REFERENCES}

[1] D. I. CARTwright, T. STEGER, Elementary symmetric polynomials in numbers of modulus 1, Canad. J. Math. 54 (2002), 239-262.

[2] P. LAKATOS, On polynomials having zeros on the unit circle, C. R. Math. Acad. Sci. Soc. R. Can. 24 , (2), (2002), 91-96.

[3] P. LAKatos, On zeros of reciprocal polynomials, Publ. Math. Debrecen 61, (2002), 645-661.

[4] P. LAKatos, L. LosonCZI, Self-inversive polynomials whose zeros are on the unit circle, Publ. Math. Debrecen 65, (2004), 409-420.

[5] P. Lakatos, L. LosoncZi, On zeros of reciprocal polynomials of odd degree, J. Inequal. Pure Appl. Math. 4 (3) (2003) Article 60, 8 pp. (electronic, http://jipam.vu.edu.au).

[6] D. S. Mitrinović, Analytic inequalities, Springer Verlag, Berlin-Heidelberg-New York, 1970.

[7] C. P. NiCUlESCU, Interpolating Newton's ineuqalities, Bull. Math. Soc. Sc. Math. Roumanie 47, (95) (2004), 67-83.

[8] T. SzELE, Introduction to Algebra. (in Hungarian) Tankönyvkiadó, Budapest 1955.

[9] A. SCHINZEL, Self-inversive polynomials with all zeros on the unit circle, Ramanujan Journal, 9, (2005), 19-23. 